\title{
Role of microRNA-141 in colorectal cancer with lymph node metastasis
}

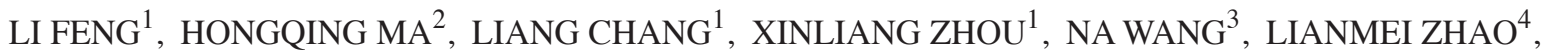 \\ JING ZUO $^{1}$, YUDONG WANG ${ }^{1}$, JING HAN $^{1}$ and GUIYING WANG ${ }^{2}$ \\ ${ }^{1}$ Department of Medical Oncology; ${ }^{2}$ Second Department of General Surgery; ${ }^{3}$ Department of Molecular Biology; \\ ${ }^{4}$ Research Center, The Fourth Hospital of Hebei Medical University, Shijiazhuang, Hebei 050011, P.R. China
}

Received March 3, 2015; Accepted May 19, 2016

DOI: $10.3892 / \mathrm{etm} .2016 .3751$

\begin{abstract}
The present study aimed to investigate the role of microRNA (miR)-141 in the pathogenesis of colorectal cancer (CRC). In total, $58 \mathrm{CRC}$ patients were included in the present study. The mRNA and protein expression levels of mitogen-activated protein kinase 4 (MAP4K4) were detected by reverse transcription-quantitative polymerase chain reaction (RT-qPCR) and western blot analysis, respectively. The miRNA-141 expression was measured by RT-qPCR, while serum MAP4K4 content was detected by enzyme-linked immunosorbent assay. Natural killer (NK) cells and T cells in peripheral blood were detected by flow cytometry. The results indicated that the mRNA and protein expression levels of MAP4K4 were significantly elevated in the tumor tissues, lymph nodes $(\mathrm{P}<0.01)$ and serum $(\mathrm{P}<0.05)$ in $\mathrm{CRC}$. Furthermore, the expression levels of MAP4K4 in CRC patients with lymph node metastasis were higher compared with those in patients without metastasis. Bioinformatics analysis revealed that MAP4K4 may be the target gene of miRNA-141. The expression levels of miRNA-141 in the tumor tissues, lymph nodes and serum were significantly decreased in CRC patients, with a more evident decline in cases with lymph node metastasis. In addition, the percentage of $\mathrm{NK}, \mathrm{CD}^{+} \mathrm{T}$ and $\mathrm{CD}^{+} \mathrm{T}$ cells was significantly decreased, whilst the number of $\mathrm{CD}^{+} \mathrm{T}$ cells was significantly increased, in the peripheral blood in CRC. The present results showed that miRNA-141 was downregulated in $\mathrm{CRC}$, which increased the expression levels of MAP4K4 and altered the anti-tumor response, further increasing the proliferation, invasion and metastasis of the tumors. These findings may contribute to improving the current understanding of the pathogenesis of CRC, and lead to the development of therapies involving miRNA-141.
\end{abstract}

Correspondence to: Dr Guiying Wang, Second Department of General Surgery, The Fourth Hospital of Hebei Medical University, 12 Jiankang Road, Shijiazhuang, Hebei 050011, P.R. China E-mail:wgy777@yeah.net

Key words: colorectal cancer, lymph node metastasis, microRNA, mitogen activated protein kinase 4

\section{Introduction}

In China, the incidence and mortality rates of colorectal cancer (CRC) were 23.03/100,000 and 11.11/100,000, respectively, in 2011, ranking only after lung cancer and gastric cancer (1). As a result of early stage CRC not displaying typical symptoms and signs associated with the disease, patients are predominantly diagnosed at an advanced stage, often accompanied by metastasis, thus missing the optimal time-frame for effective treatment (2). In general, lymph node metastasis represents the first step of tumor dissemination for CRC, and lymph node micrometastasis is currently an accurate indicator for the clinical staging, treatment and prognostic determination of CRC (3). If the regional lymph nodes were not appropriately treated, the traditional radical lumpectomy of CRC alone may lead to inadequate or excessive chemotherapy, which may cause unwanted damage to normal organs and tissues. Therefore, lymph node metastasis in CRC has become an important research focus for the elucidation of disease pathogenesis and treatment, while the identification of the regulating factors involved has also attracted increasing attention.

The pathogenesis and development of CRC have been found to be regulated by various signaling pathways and other factors. For instance, it has been shown that homolog 8 (CBX8) and insulin-like growth factor-1 (IGF1) are closely associated with the onset of CRC (4), and microRNA (miRNA)-92 is a key oncogene in the development of the disease (5). Conversely, the status of the body's immune system is also important for tumor proliferation and metastasis. The anti-tumor response is predominantly achieved by cellular immunity, and natural killer (NK) cells and T-lymphocyte subsets have been found to be implicated in tumor immune surveillance (6).

Mitogen-activated protein kinase kinase kinase kinase 4 (MAP4K4) is an upstream activator in the MAPK signaling pathway, which has been demonstrated to promote the invasion, metastasis and development of ovarian, breast and prostate cancer, and malignant melanoma (7-11). MAP4K4 has been demonstrated to be overexpressed in various tumors, accelerating tumor cell transformation, promoting cell invasion and decreasing cell adhesion (12). Furthermore, the expression of MAP4K4 in CRC without lymph node metastasis is significantly lower compared with lymph node metastasis, indicating the role of MAP4K4 in promoting CRC proliferation, invasion 
and metastasis (13). However, the effect of MAP4K4 on the immune system in CRC has yet to be fully elucidated.

In the present study, the role of miRNA-141 in the pathogenesis of $\mathrm{CRC}$, especially concerning its regulation of MAP4K4, was investigated for the first time. The expression levels of MAP4K4 and miRNA-141 in the tumor, lymph nodes and serum of CRC patients were detected and analyzed, while the immune system in CRC was also evaluated.

\section{Patients and methods}

Patients. In total, 58 patients with CRC were included in the present study, who had been diagnosed and subjected to total surgical resection prior to radiotherapy at The Fourth Hospital of Hebei Medical University (Shijiazhuang, China) between January 2014 and December 2014. Disease diagnosis was based on the clinical presentation, medical history and family history, physical examination, laboratory tests, endoscopy, imaging detection, and histopathological examination (14). Of these patients, there were 5 cases of tubulovillous adenoma, 11 cases of papillary carcinoma, 21 cases of tubular adenocarcinoma, 13 cases of mucinous adenocarcinoma, 6 cases of signet ring cell carcinoma and 2 cases of undifferentiated carcinoma. The occurrence of lymph node metastasis was confirmed in 26 cases by postoperative pathological examination on biopsy, and the remaining 32 cases were free from lymph node metastasis. All patients were first-onset cases and had not previously received hormone therapy, radiotherapy or chemotherapy prior to surgery. In addition, 29 age- and gender-matched healthy individuals were enrolled into the control group. The CRC patients with lymph node metastasis included 11 males and 15 females, with ages of 34-85 years (median age, 61 years). CRC patients without lymph node metastasis included 17 males and 15 females, aged between 28-76 years (median age, 58 years). In the control group, there were 13 males and 16 females, aged from $22-72$ years (median age, 55 years). Prior written and informed consent was obtained from each patient and the study was approved by the Ethics Review Board of the Fourth Hospital of Hebei Medical University.

Sample collection. The following specimens were collected from the subjects in the present study: i) The excised tumor and adjacent tissues, which were stored in liquid nitrogen; ii) the lymph nodes in proximity to the surgical site, which were removed during surgery and stored in liquid nitrogen; and iii) the peripheral blood collected under fasting conditions in the morning, which was mixed with EDTA anticoagulant (cat. no. 367525; BD Biosciences, San Jose, CA, USA) and stored at $-20^{\circ} \mathrm{C}$.

Reverse transcription-quantitative polymerase chain reaction (RT-qPCR). The mRNA expression levels of MAP4K4 and miRNA-141 were detected with the use of RT-qPCR. Briefly, for detection of the expression levels in tumor and in lymph nodes, total RNA was extracted with TRIzol reagent (Invitrogen; Thermo Fisher Scientific, Inc., Waltham, MA, USA). The serum RNA was extracted with the miRNeasy Serum/Plasma kit (Guangzhou Jianlun Biological Technology Co., Ltd., Guangzhou, China). RT was performed to obtain the cDNA with the RevertAid First Strand cDNA Synthesis kit (Fermentas; Thermo Fisher Scientific, Inc.), according to the manufacturer's protocol. The $25 \mu \mathrm{l}$ PCR system contained 2 liters template, 1 liter each primer, 12.5 liters TransStart Top Green qPCR SuperMix (Transgen Biotech, Inc., Beijing, China), and 8.5 liter double distilled $\mathrm{H}_{2} \mathrm{O}$.

For the detection of MAP4K4, the primer sequences were as follows: MAP4K4 forward, 5'-AAGGAGAGAGCGGGAAGC TA-3', and reverse, 5'-TTGTTGCAACTGCCTCTGGA-3'; GAPDH forward, 5'-GTTGGAGGTCGGAGTCAACGGA-3', and reverse, 5'-GAGGGATCTCGCTCCTGGAGGA-3'. The PCR conditions consisted of denaturation at $94^{\circ} \mathrm{C}$ for $5 \mathrm{~min}$, followed by $94^{\circ} \mathrm{C}$ for $30 \mathrm{sec}, 60^{\circ} \mathrm{C}$ for $30 \mathrm{sec}$ and $72^{\circ} \mathrm{C}$ for $45 \mathrm{sec}$, for a total of 35 cycles. For the detection of miRNA-141, the primer sequences were as follows: miRNA-141, 5'-CCG GTAACACTGTCTGGTAA-3', and U6, 5'-GCTTCGGCA GCACATATACTAAAAT-3'. The PCR conditions were as follows: Denaturation at $95^{\circ} \mathrm{C}$ for $5 \mathrm{~min}$, followed by $95^{\circ} \mathrm{C}$ for $15 \mathrm{sec}, 58^{\circ} \mathrm{C}$ for $30 \mathrm{sec}$ and $72^{\circ} \mathrm{C}$ for $30 \mathrm{sec}$, for 40 cycles. The relative expression levels of target genes were calculated with the $2^{-\Delta \Delta \mathrm{Cq}}$ method (15).

Western blot analysis. Normal, tumor and lymph node metastasis tissues were lysed on ice with lysis buffer (Beyotime Institute of Biotechnology, Haimen, China), and the protein concentration was determined with a BCA kit (cat. no. P0009; Beyotime Institute of Biotechnology). Protein samples (20 mg) were subjected to $10 \%$ sodium dodecyl sulfate-polyacrylamide gel electrophoresis, and then electronically transferred onto a nitrocellulose membrane. The blot was blocked with 5\% non-fat milk at room temperature for $1 \mathrm{~h}$, and then incubated with rabbit anti-human anti-MAP4K4 polyclonal primary antibody (1:1,000; cat. no. ab155583; Abcam, Cambridge, MA, USA) or rabbit anti-human anti-GAPDH polyclonal primary antibody (1:5,000; cat. no. ab9485; Abcam) at $4^{\circ} \mathrm{C}$ overnight. The membrane was then incubated with goat anti-rabbit immunoglobulin G (1:3,000; cat. no. ab6721; Abcam) at room temperature for $1 \mathrm{~h}$. Subsequently, the blot was developed using an enhanced chemiluminescence system (cat. no. P0018A; Beyotime Institute of Biotechnology), and the protein bands were analyzed with the Image Lab software version 3.0 (Bio-Rad Laboratories, Inc., Hercules, CA, USA).

Enzyme-linked immunosorbent assay (ELISA). Serum MAP4K4 contents were detected with an ELISA kit (cat. no. CSB-EL013439HU; Weijia Technology Co., Ltd., Guangzhou, China) according to the manufacturer's instructions. Blood sample was centrifuged at $4^{\circ} \mathrm{C}$ at $800 \mathrm{x}$ g for $10 \mathrm{~min}$, separating the serum from the blood cells. Sample $(10 \mu \mathrm{l})$ was then added into the 96 -well plates in the ELISA microplate, followed by the addition of $40 \mu \mathrm{l}$ diluting solution. The experiment was performed in triplicate. Subsequently, $100 \mu 1$ horseradish peroxidase-labeled antibody (contained within the ELISA kit) was added into each well, and the plate was placed in an incubator for $1 \mathrm{~h}$. After washing five times with $\mathrm{ddH}_{2} \mathrm{O}, 50 \mu \mathrm{l}$ substrate $\mathrm{A}$ and $50 \mu \mathrm{l}$ substrate $\mathrm{B}$ were added into each well, respectively, and the plate was incubated at $37^{\circ} \mathrm{C}$ for $15 \mathrm{~min}$. The reaction was stopped by adding $50 \mu \mathrm{l}$ stopping solution, and the optical density at $450 \mathrm{~nm}$ was read on a microplate reader within $15 \mathrm{~min}$. 
Flow cytometry. NK and T cells in the peripheral blood were detected by flow cytometric analysis with $\mathrm{CD} 3 / \mathrm{CD} 4 / \mathrm{CD} 8$ and CD3/CD16+56 agents (cat. nos. IM1650 and IM2076, respectively; Immunotech; Beckman Coulter, Inc., Marseille, France), according to the manufacturer's instructions. Briefly, $20 \mathrm{ml}$ staining agent was added into the anticoagulated whole blood at room temperature for $25 \mathrm{~min}$, and $2 \mathrm{ml}$ hemolytic agent was then added at room temperature for $10 \mathrm{~min}$. Following centrifugation at $450 \mathrm{xg}$ at $4^{\circ} \mathrm{C}$ for $5 \mathrm{~min}$, the supernatant was discarded. After washing with phosphate-buffered saline twice, $500 \mu 11 \%$ paraformaldehyde was added for fixation. The sample was detected with a FACScan flow cytometer (BD Biosciences), and the data were analyzed using Cellquest software version 3.1 (BD Biosciences).

Bioinformatics analysis. To determine the target gene of miRNA-141, bioinformatics analysis was performed with the following online software and/or websites: miRanda (http:// www.microrna.org/microrna/getExprForm.do), TargetScan (www.targetscan.org), PiTa (http://genie.weizmann.ac.il/pubs/ mir07/mir07_data.html), RNAhybrid (http://bibiserv.techfak. uni-bielefeld.de/rnahybrid/) and PICTA (http://pictar.mdcberlin.de/).

Statistical analysis. Data are expressed as the mean \pm standard deviation. SPSS software (version 18.0; SPSS, Inc., Chicago, IL, USA) was used for statistical analysis. One-way analysis of variance was performed for the comparison between the groups, along with the least-significant difference and Student-Newman-Keuls tests (for equal variance), or the Tamhane's T2 and Dunnett's T3 tests (when equal variance was not assumed). $\mathrm{P}<0.05$ was considered to indicate a statistically significant difference.

\section{Results}

mRNA expression levels of MAP4K4 are elevated in the tumor tissues, lymph nodes and serum in CRC patients. In order to investigate the mRNA expression levels of MAP4K4 in the tumor tissues, lymph nodes and serum in CRC patients with or without lymph node metastasis, RT-qPCR was performed. The present results indicated that, compared with the control group, the mRNA expression levels of MAP4K4 were significantly elevated in the tumor tissues, lymph nodes and serum in patients with CRC (all $\mathrm{P}<0.05$; Fig. 1). Furthermore, within the CRC patients, the mRNA expression levels of MAP4K4 in the tumor tissues, lymph nodes and serum in cases with lymph node metastasis were all significantly higher compared with those in cases without lymph node metastasis $(\mathrm{P}<0.05$; Fig. 1). Thus, the results suggest that MAP4K4 may be upregulated in CRC, particularly in cases with lymph node metastasis, and this may contribute to the disease pathogenesis.

Protein expression levels of MAP4K4 are increased in the tumor tissues, lymph nodes and serum in CRC patients. The protein expression levels of MAP4K4 in the tumor tissues and lymph nodes were detected by western blot analysis, while the serum expression was detected by ELISA. The results of western blot analysis indicated that, compared with the control group, the protein expression levels of MAP4K4 were

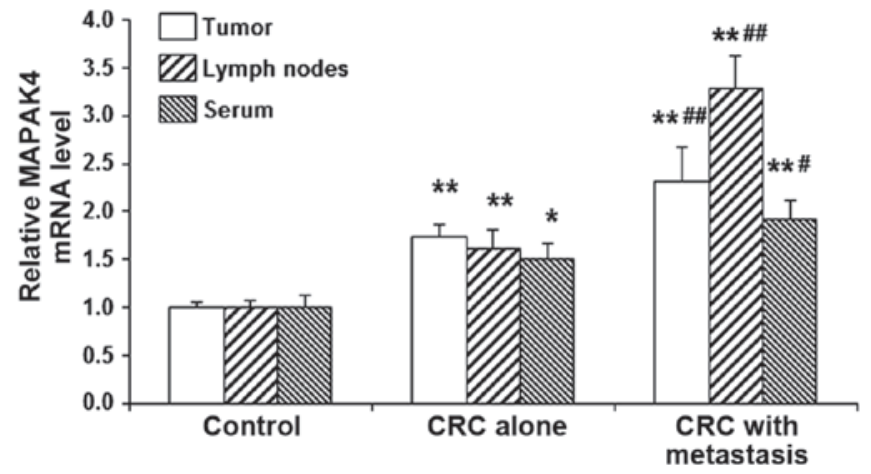

Figure 1. mRNA expression levels of MAP4K4 were elevated in CRC. The mRNA expression levels of MAP4K4 in the tumor, lymph nodes and serum in CRC with or without lymph node metastasis (CRC with metastasis and CRC-alone groups, respectively) were detected with reverse transcriptionquantitative polymerase chain reaction. ${ }^{*} \mathrm{P}<0.05$ and ${ }^{* *} \mathrm{P}<0.01$ vs. control group; ${ }^{\#} \mathrm{P}<0.05$ and ${ }^{\# \#} \mathrm{P}<0.01$ vs. $\mathrm{CRC}$-alone group. $\mathrm{CRC}$, colorectal cancer; MAP4K4, mitogen-activated protein kinase kinase kinase kinase 4.

significantly increased in $\mathrm{CRC}$ tissues $(\mathrm{P}<0.05)$ and more evidently in the lymph nodes ( $\mathrm{P}<0.01$; Fig. $2 \mathrm{~A}$ and $\mathrm{B})$. In addition, the protein expression levels of MAP4K4 in the tumor and lymph nodes in CRC patients with lymph node metastasis were all significantly higher compared with those without lymph node metastasis ( $\mathrm{P}<0.05$; Fig. $2 \mathrm{~A}$ and $\mathrm{B})$.

Similar results were obtained for the protein expression of MAP4K4 in the serum. ELISA analysis revealed that, compared with the control group, the serum MAP4K4 expression was significantly increased in CRC patients with and without lymph node metastasis $(\mathrm{P}<0.01)$. However, the expression was elevated to a greater extent in the cases with lymph node metastasis compared with cases without metastasis $(\mathrm{P}<0.01$; Fig. $2 \mathrm{C})$. In accordance with the alteration of the mRNA expression levels of MAP4K4, the aforementioned results indicated that MAP4K4 may be involved in the pathogenesis of CRC, particularly in the development of metastasis via the lymphatic and blood circulation.

Expression levels of miRNA-141 are reduced in the tumor tissues, lymph nodes and serum in CRC patients. It has been reported that MAP4K4 may be regulated by miRNA-141 in cases of pancreatic cancer (16). To further investigate the underlying mechanisms through which MAP4K4 is modulated in CRC, bioinformatics analysis was performed. Results from the miRanda, TargetSean, PiTa, RNAhybrid, and PICTA analyses revealed that MAP4K4 may be the target gene of miRNA-141 (Fig. 3), and may be involved in the pathogenesis of CRC. RT-qPCR was then performed to detect the expression levels of miRNA-141 in the tumor tissues, lymph nodes and serum from CRC patients. The current results demonstrated that, compared with the control group, the expression levels of miRNA-141 in the tumor tissues, lymph nodes and serum were significantly decreased in CRC patients $(\mathrm{P}<0.05$; Fig. 4). Furthermore, of the CRC patients, the expression levels of miRNA-141 in the tumor tissues, lymph nodes and serum in cases with lymph node metastasis decreased to a significant extent when compared with those in patients without lymph node metastasis $(\mathrm{P}<0.05$; Fig. 4). The results suggest that miRNA-141 may participate 
A
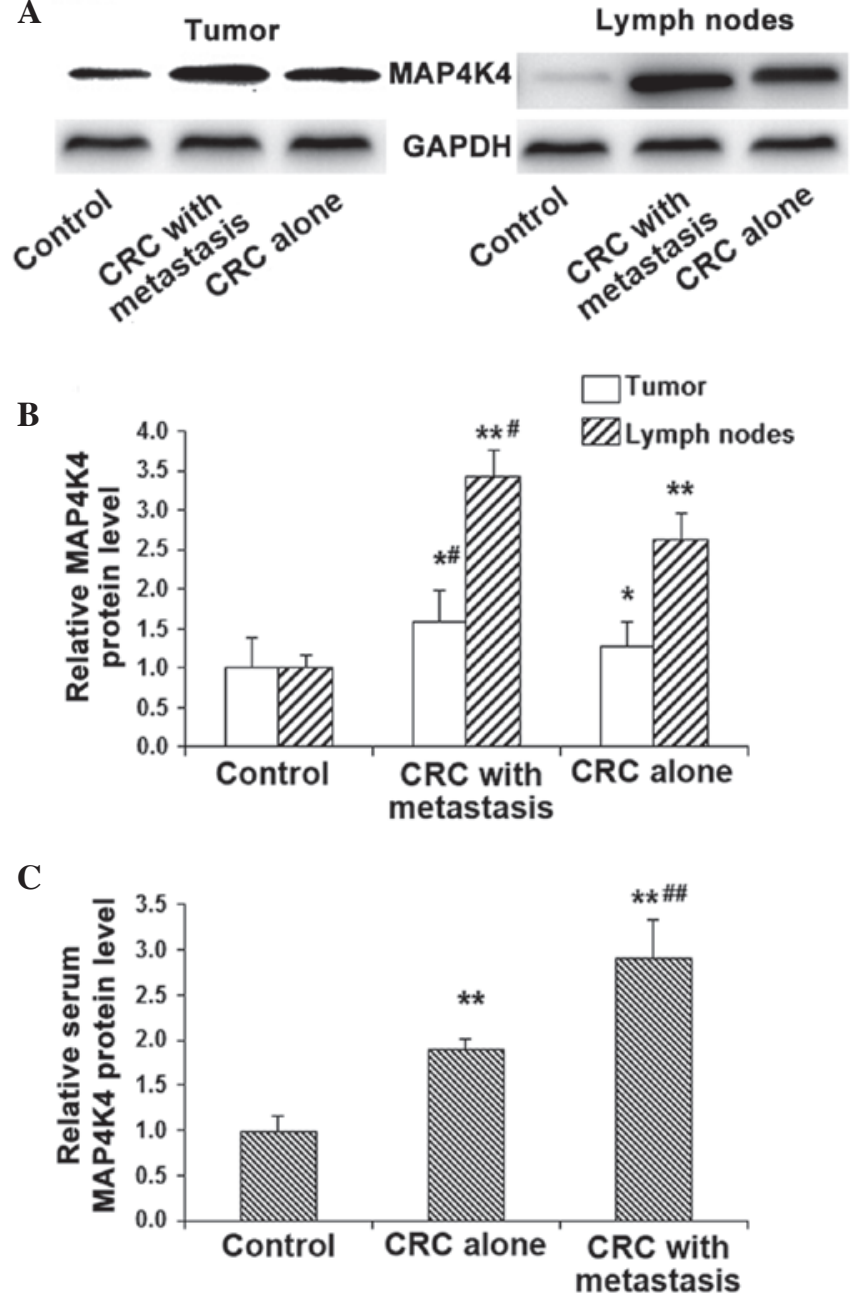

Figure 2. Protein expression levels of MAP4K4 were increased in CRC. (A) Western blot analysis performed to detect the protein expression levels of MAP4K4 in tumor and lymph nodes in CRC with or without lymph node metastasis (CRC with metastasis and CRC-alone groups, respectively). (B) Statistical analysis of the expression levels of MAP4K4 in tumor and lymph nodes. (C) Expression levels of MAP4K4 in the serum were detected with ELISA. $P<0.05$ and ${ }^{* *} \mathrm{P}<0.01$ vs. the control group; ${ }^{*} \mathrm{P}<0.05$ and ${ }^{\# \#} \mathrm{P}<0.01$ vs. the CRC-alone group. CRC, colorectal cancer; MAP4K4, mitogen-activated protein kinase kinase kinase kinase 4 .

in the pathogenesis and development of CRC, and contribute to lymph node metastasis.

$N K$ and T-cell subsets are changed in peripheral blood in CRC. To investigate the anti-tumor response in CRC, the NK cells and T-lymphocyte subsets were detected with flow cytometry. The results revealed that, compared with the control group, the percentage of $\mathrm{NK}, \mathrm{CD}^{+} \mathrm{T}$ and $\mathrm{CD}^{+} \mathrm{T}$ cells in the peripheral blood of CRC patients were significantly decreased, while the $\mathrm{CD} 8^{+} \mathrm{T}$ cells were significantly increased $(\mathrm{P}<0.05$ and $\mathrm{P}<0.01$; Fig. $5 \mathrm{~A})$, resulting in significantly decreased $\mathrm{CD} 4^{+} / \mathrm{CD} 8^{+}$ratios $(\mathrm{P}<0.01$; Fig. 5B). Furthermore, the alterations were significantly more marked in the CRC patients with lymph node metastasis compared with those without lymph node metastasis $(\mathrm{P}<0.05)$. Thus, the results reveal declined anti-tumor responses in CRC patients, which is worse in cases with lymph node metastasis compared with those without metastasis.
3' gguagaaaUGGUCUGUCACAAu 5' has-miRNA-141 | $|\vdots \vdots \quad|||||||$ 1291: 5' ucacacaaACUGUUCAGUCUUg 3' MAP4K4

Figure 3. Bioinformatics analysis predicted that miRNA-141 targeted on MAP4K4. MAP4K4, mitogen-activated protein kinase kinase kinase kinase 4; miRNA, microRNA.

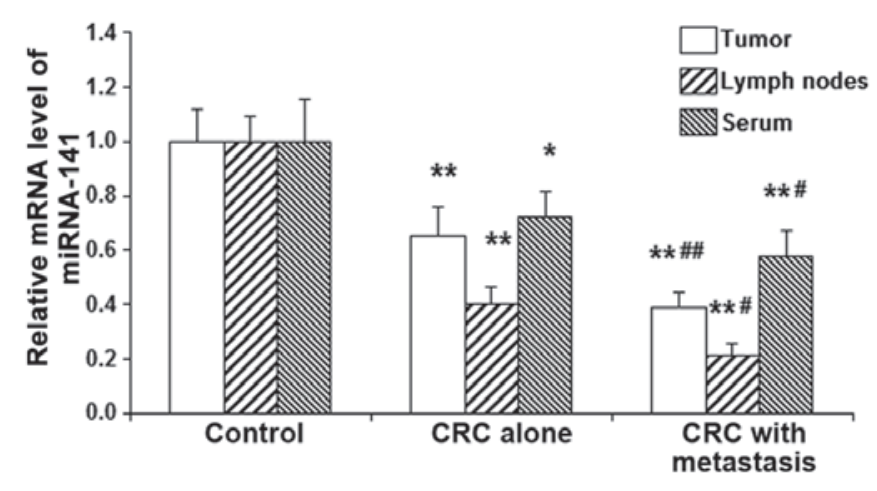

Figure 4. Expression levels of miRNA-141 were declined in CRC. The expression levels of miRNA-141 in the tumor, lymph nodes and serum in CRC with or without lymph node metastasis (the CRC with metastasis group and the CRC-alone group, respectively) were detected with reverse transcription-quantitative polymerase chain reaction. ${ }^{*} \mathrm{P}<0.05$ and ${ }^{* *} \mathrm{P}<0.01$ vs. the control group; ${ }^{\#} \mathrm{P}<0.05$ and ${ }^{\# \#} \mathrm{P}<0.01$ vs. $\mathrm{CRC}$-alone group. miRNA, microRNA; CRC, colorectal cancer.

\section{Discussion}

In the present study, the expression levels of MAP4K4 and the upstream miRNA-141 in the tumor tissues, lymph nodes and serum in patients with CRC with or without lymph node metastasis were investigated. In addition, the anti-tumor responses (as indicated by the NK cells and T-lymphocyte subsets) were also detected. The underlying mechanisms for the lymph node metastasis of CRC and the changes in the immune system, particularly those concerning the regulation of MAP4K4 by miRNA-141, were also discussed.

In 2011, >12 million cases were diagnosed with new-onset CRC worldwide, ranking third in terms of incidence in cases of malignant tumors in males, and only second to breast cancer in females $(17,18)$. The primary treatment for CRC is surgical resection, combined with radiotherapy and chemotherapy, as well as molecular targeted therapy. However, the surgical cure rates and the postoperative survival rates are not satisfactory $(19,20)$. The 5 -year survival rate of CRC is associated with the local infiltration and regional lymph node metastasis. According to the American Joint Committee on Cancer (AJCC), CRC may be divided into stages II and III, based on the presence or absence of regional lymph node metastasis (21). Dukes' staging (stages B and C) is also associated with the status of regional lymph node metastasis (22). Therefore, it is of great importance to screen for lymph node metastasis and to investigate the early-diagnosis molecular indicators for CRC.

The role of MAP4K4 in the pathogenesis of CRC was investigated in the present study. Dhillon et al (23) reported that MAP4K4 was able to activate $\mathrm{p} 38$ stress-activated protein kinase to enhance tumor proliferation. Wright et al (12) indicated that MAP4K4 was able to promote the malignant transformation, 
$\mathbf{A}$
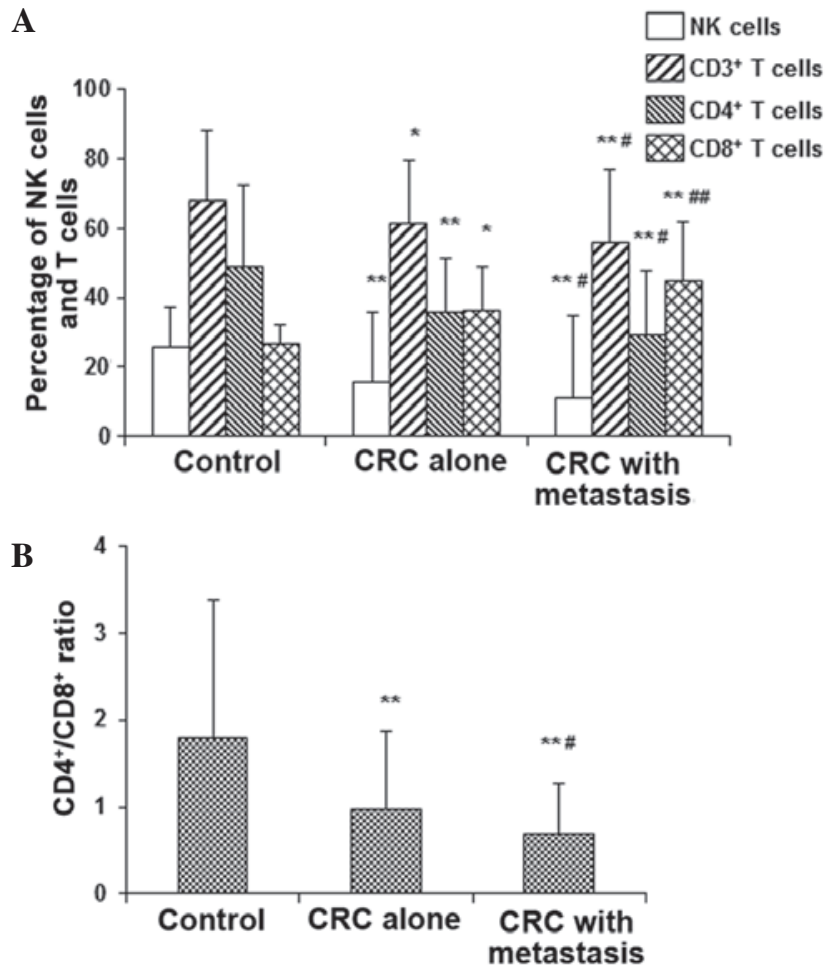

Figure 5. NK cells and T cell subsets were altered in CRC. The NK cells and T-lymphocyte subsets in peripheral blood in CRC with or without lymph node metastasis (CRC with metastasis and CRC-alone groups, respectively) were detected with flow cytometry. (A) Statistical analysis of the NK cells, $\mathrm{CD}^{+}, \mathrm{CD}^{+}$, and $\mathrm{CD} 8^{+} \mathrm{T}$ cells in the peripheral blood in CRC. (B) Statistical analysis of the $\mathrm{CD} 4^{+} / \mathrm{CD} 8^{+}$ratios in $\mathrm{CRC}$. ${ }^{*} \mathrm{P}<0.05$ and ${ }^{* *} \mathrm{P}<0.01$ vs. the control group; ${ }^{\#} \mathrm{P}<0.05$ and ${ }^{\# \#} \mathrm{P}<0.01$ vs. the $\mathrm{CRC}$-alone group. $\mathrm{CRC}$, colorectal cancer; NK, natural killer.

the colony formation and increase cell invasion and metastasis. In addition, using siRNA technology, Collins et al (11) demonstrated that the knockdown of MAP4K4 was able to inhibit the invasion of ovarian cancer cells. In accordance with the aforementioned findings, the results of the current study showed that the expression levels of MAP4K4 were significantly elevated in the tumor and lymph nodes in CRC, indicating that MAP4K4 may promote the pathogenesis and development of tumors, and regulate tumor proliferation and invasion. Furthermore, the mRNA and protein expression levels of MAP4K4 in the serum were also significantly elevated in CRC patients. As the blood circulation is an important factor for tumor metastasis, the current results suggest that MAP4K4 may contribute to the metastasis of CRC via the blood circulation.

miRNA is able to regulate the mRNAs of target genes that may serve an important role in the development and progression of tumors $(24,25)$. It has been reported that a class of endogenous, small non-coding miRNAs may act upon the mRNA of MAP4K4 and inhibit its translation (26). To further evaluate the regulating mechanisms of MAP4K4 in CRC, bioinformatics analysis was performed. The results revealed that miRNA-141, which has previously been confirmed to serve a role in the occurrence and development of pancreatic cancer (16), may be the upstream regulator for MAP4K4 in CRC. Furthermore, compared with CRC patients without lymph node metastasis, the expression levels of miRNA-141 were significantly higher in the tumor tissues, lymph nodes, and serum in cases with lymph node metastasis. These results suggest that the downregulation of miRNA-141 may be associated with the proliferation, infiltration and metastasis of CRC. According to the present results concerning MAP4K4 in CRC, the upregulation of MAP4K4 may be associated with the downregulation of miRNA-141 in the development and progression of CRC. Considering the association between miRNA-141 and MAP4K4, as well as the important role of MAP4K4 in tumorigenesis, miRNA-141 expression (particularly in the serum) may be used as an indicator of CRC metastasis. The regional lymph node metastasis of CRC is known to result from the local tumor invasion; in addition, distant metastasis and/or metastasis into other organs is also observed $(27,28)$. Accordingly, the downregulation of miRNA-141 may also contribute to the tumor dissemination into other organs and tissues.

In order to investigate the effects of miRNA-141 on the immune system, the NK and T cells in the peripheral blood of CRC patients were detected. The results demonstrated that the percentages of $\mathrm{CD}^{+}$and $\mathrm{CD} 4^{+} \mathrm{T}$ cells were significantly decreased, whereas the percentage of $\mathrm{CD}^{+} \mathrm{T}$ cells was significantly increased in CRC patients, resulting in a decreased $\mathrm{CD} 4^{+} / \mathrm{CD} 8^{+}$ratio. Changes in the immune system were more evident in cases with lymph node metastasis, compared with the CRC patients without lymph node metastasis. NK cells and T lymphocytes represent the initial defense against tumors, thus, their dysfunction would weaken tumor resistance. The elevation in CD8 may result from the T lymphocytes induced by the tumor-secreted immunosuppressive factors $(29,30)$, which may disturb the immune surveillance and facilitate the proliferation and metastasis of tumors. Therefore, these results suggest that the alterations in the immune system may be associated with the changes in miRNA-141 and MAP4K4 expression levels, which may contribute to the pathogenesis of CRC. Considering the various factors associated with the pathogenesis of CRC (31-33), and due to the limited number of subjects enrolled in the present study, further in-depth studies are required to investigate the detailed mechanisms through which miRNA-141 and MAP4K4 are involved in the development of CRC.

In conclusion, the current results showed that the mRNA and protein expression levels of MAP4K4 were elevated in the tumor tissues, lymph nodes and serum of CRC patients. The expression levels of MAP4K4 were higher in CRC patients with lymph node metastasis compared with those in patients without metastasis. Furthermore, the expression levels of miRNA-141 were reduced in the tumor tissues, lymph nodes and serum in CRC, with a more evident decline observed in cases with lymph node metastasis. In addition, the percentage of NK, $\mathrm{CD}^{+} \mathrm{T}$, and $\mathrm{CD} 4^{+} \mathrm{T}$ cells was significantly decreased and that of $\mathrm{CD}^{+} \mathrm{T}$ cells was significantly increased in the peripheral blood of the CRC patients, resulting in significantly decreased $\mathrm{CD}^{+} / \mathrm{CD}^{+}+$ratios. The aforementioned findings may contribute to an improved understanding of the pathogenesis of CRC and provide evidence for the application of therapies involving miRNA-141.

\section{Acknowledgements}

The present study was supported by the Key Projects of Medical Sciences from the Hebei Provincial Department of Health (grant no. 20150769). 


\section{References}

1. Wan DS: Epidemiological trend and control strategy of colorectal cancer in China. Zhong Hua Zhong Liu Za Zhi 33: 481-483, 2011 (In Chinese).

2. Thosani N, Guha S and Singh H: Colonoscopy and colorectal cancer incidence and mortality. Gastroenterol Clin North Am 42 619-637, 2013

3. Wang Y, Chou WP, Shen CJ, Wang XY and Luo J: Risk factors of postoperative recurrence after radical resection of colon cancer. Dalian Yi Ke Da Xue Xue Bao 35: 65-67, 2013 (In Chinese).

4. Yang S, Liu W, Li M, Wen J, Zhu M and Xu S: Insulin-like growth factor-1 modulates polycomb Cbx8 expression and inhibits colon cancer cell apoptosis. Cell Biochem Biophys Nov 15, 2014 (Epub ahead of print).

5. Tsuchida A, Ohno S, Wu W, Borjigin N, Fujita K, Aoki T, Ueda S, Takanashi M and Kuroda M: miR-92 is a key oncogenic component of the miR-17-92 cluster in colon cancer. Cancer Sci 102: 2264-2271, 2011

6. Väyrynen JP, Kantola T, Väyrynen SA, Klintrup K, Bloigu R, Karhu T, Mäkelä J, Herzig KH, Karttunen TJ, Tuomisto A and Mäkinen MJ: The relationships between serum cytokine levels and tumor infiltrating immune cells and their clinical significance in colorectal cancer. Int J Cancer 139: 112-121, 2016.

7. Kim JM, Cho SJ, OH YK, Jung HY, Kim YJ and Kim N: Nuclear factor-kappa B activation pathway in intestinal epithelial cells is a major regulator of chemokine gene expression and neutrophil migration induced by Bacteroides fragilis enterotoxin. Clin Exp Immunol 130: 59-66, 2002.

8. Jin SH, Kim TI, Han DS, Shin SK and Kim WH: Thalidomide suppresses the interleukin 1beta-induced NFkappaB signaling pathway in colon cancer cells. Ann N Y Acad Sci 973: 414-418, 2002.

9. Royuela M, Rodríguez-Berriguete G, Fraile B and Paniagua R: TNF-alpha/IL-1/NF-kappaB transduction pathway in human cancer prostate. Histol Histopathol 23: 1279-1290, 2008.

10. Rangaswami $\mathrm{H}$ and Kundu GC: Osteopontin stimulates melanoma growth and lung metastasis through NIK/MEKK1-dependent MMP-9 activation pathways. Oncol Rep 18: 909-915, 2007.

11. Collins CS, Hong J, Sapinoso L, Zhou Y, Liu Z, Micklash K, Schultz PG and Hampton GM: A small interfering RNA screen for modulators of tumor cell motility identifies MAP4K4 as a promigratory kinase. Proc Natl Acad Sci USA 103: 3775-3780, 2006.

12. Wright JH, Wang X, Manning G, LaMere BJ, Le P, Zhu S, Khatry D, Flanagan PM, Buckley SD, Whyte DB, et al: The STE20 kinase HGK is broadly expressed in human tumor cells and can modulate cellular transformation, invasion and adhesion. Mol Cell Biol 23: 2068-2082, 2003.

13. Wang B, Shen ZL, Gao ZD, Zhao G, Wang CY, Yang Y, Zhang JZ, Yan YC, Shen C, Jiang KW, et al: MiR-194, commonly repressed in colorectal cancer, suppresses tumor growth by regulating the MAP4K4/c-Jun/MDM2 signaling pathway. Cell cycle 14: 1046-1058, 2015.

14. Bureau of Medical Administration, National Health; Oncology Branch of Chinese Medical Association: Standardization of diagnosis and treatment for colorectal cancer in China (2015 edition). Zhonghua Xiaohua Waike Zazhi 14: 783-799, 2015 (In Chinese).

15. Grimholt RM, Urdal P, Klingenberg O and Piehler AP: Rapid and reliable detection of $\alpha$-globin copy number variations by quantitative real-time PCR. BMC Hematol 14: 4, 2014.
16. Zhao G, Wang B, Liu Y, Zhang JG, Deng SC, Qin Q, Tian K, Li X, Zhu S, Niu Y, et al: miRNA-141, downregulated in pancreatic cancer, inhibits cell proliferation and invasion by directly targeting MAP4K4. Mol Cancer Ther 12: 2569-2580, 2013.

17. Jemal A, Siegel R, Xu J and Ward E: Cancer statistics, 2010. CA Cancer J Clin 60: 277-300, 2010

18. Jemal A, Bray F, Center MM, Ferlay J, Ward E and Forman D: Global cancer statistics. CA Cancer J Clin 61: 69-90, 2011.

19. Edwards BK, Ward E, Kohler BA, Eheman C, Zauber AG, Anderson RN, Jemal A, Schymura MJ, Lansdorp-Vogelaar I, Seeff LC, et al: Annual report to the nation on the status of cancer, 1975-2006, featuring colorectal cancer trends and impact of interventions (risk factors, screening and treatment) to reduce future rates. Cancer 116: 544-573, 2010.

20. Franko J, Shi Q, Goldman CD, Pockaj BA, Nelson GD, Goldberg RM, Pitot HC, Grothey A, Alberts SR and Sargent DJ: Treatment of colorectal peritoneal carcinomatosis with systemic chemotherapy: A pooled analysis of north central cancer treatment group phase III trials N9741 and N9841. J Clin Oncol 30: 263-267, 2012.

21. Edge SB and Compton CC: The American joint committee on cancer: The 7th edition of the AJCC cancer staging manual and the future of TNM. Ann Surg Oncol 17: 1471-1474, 2010.

22. Akkoca AN, Yanik S, OzdemirZT, Cihan FG, Sayar S, Cincin TG, Cam $\mathrm{A}$ and Ozer C: TNM and modified dukes staging along with the demographic characteristics of patients with colorectal carcinoma. Int J Clin Exp Med 7: 2828-2835, 2014.

23. Dhillon AS, Hagan S, Rath $\mathrm{O}$ and Kolch W: MAP kinase signalling pathways in cancer. Oncogene 26: 3279-3290, 2007.

24. Chen K and Rajewsky N: The evolution of gene regulation by transcription factors and microRNAs. Nat Rev Gen 8: 93-103, 2007.

25. Lewis BP, Burge CB and Bartel DP: Conserved seed pairing, often flanked by adenosines, indicates that thousands of human genes are microRNA targets. Cell 120: 15-20, 2005.

26. Zhao X, Mohan R, Özcan S and Tang X: MicroRNA-30d induces insulin transcription factor MafA and insulin production by targeting mitogen-activated protein 4 kinase 4 (MAP4K4) in pancreatic $\beta$-cells. J Biol Chem 287: 31155-31164, 2012.

27. Iguchi N, Takeda Y, Sato N, Ukichi K, Katakura A, Ueda K, Narushima T, Higuchi S and Ogasawara K: The antihistamine olopatadine regulates $\mathrm{T}$ cell activation in palladium allergy. Int Immunopharmacol 35: 70-76, 2016.

28. Quan H, Fang L, Pan H, Deng Z, Gao S, Liu O, Wang Y, Hu Y, Fang $\mathrm{X}$, Yao $\mathrm{Z}$, et al: An adaptive immune response driven by mature, antigen-experienced $\mathrm{T}$ and $\mathrm{B}$ cells within the microenvironment of oral squamous cell carcinoma. Int J Cancer 138: 2952-2962, 2016

29. Jin B: Cell and molecular immunology. World Book Publishing Company, Beijing, 1995.

30. Bi A: Medical immunology. People's Publishing House, Beijing, 1996.

31. Wan LY, Deng J, Xiang XJ, Zhang L, Yu F, Chen J, Sun Z, Feng M and Xiong JP: miR-320 enhances the sensitivity of human colon cancer cells to chemoradiotherapy in vitro by targeting FOXM1. Biochem Biophys Res Commun 457: 125-132, 2015.

32. Sun Y, Xing X, Liu Q, Wang Z, Xin Y, Zhang P, Hu C and Liu Y: Hypoxia-induced autophagy reduces radiosensitivity by the HIF-1 $\alpha / \mathrm{miR}-210 / \mathrm{Bcl}-2$ pathway in colon cancer cells. Int $\mathrm{J}$ Oncol 46: 750-756, 2015.

33. Tian Y, Pan Q, Shang Y, Zhu R, Ye J, Liu Y, Zhong X, Li S, He Y, Chen L, et al: MicroRNA-200 (miR-200) Cluster Regulation by Achaete Scute-like 2 (Ascl2) impact on the epithelial-mesenchymal transition in colon cancer cells. J Biol Chem 289: 36101-36115, 2014 